\title{
Random exchange models and the distribution of wealth
}

\author{
Enrico Scalas ${ }^{\mathrm{a}}$ \\ Department of Mathematics, University of Sussex, Brighton BNl 9BH, UK \\ Received 17 May 2016 / Received in final form 15 September 2016 \\ Published online 22 December 2016
}

\begin{abstract}
I am presenting my personal point of view on what is interesting in Econophysics. In particular, I focus on random exchange models for the distribution of wealth in order to illustrate the concept of statistical equilibrium in Economics.
\end{abstract}

\section{Introduction}

In December 2007, I was reading the Financial Times in a hotel in Stockholm. There were news about problems in some hedge funds, hints of the troubles to come. For me, the years between 2008 and 2010 were a period of intense study in the hope to find viable alternatives to mainstream economic theory. These were years in which everybody was (re)reading Minsky [1] and/or Farjoun and Machover [2]. The Reykjavik Manifesto collects several contribution of this period [3]. Fast forward to April 2016: I am sitting in the Shard in London, attending a workshop on Real World Risks and Extremes and listening to a talk by J.-P. Bouchaud. He tells us that he recently met a prominent macroeconomist after the macroeconomist wrote that Macroeconomics needs reforms. Then, Bouchaud explains us that the macroeconomist has now changed his mind. The world economy has stabilised and we are back to business as usual. To explain this outcome, instead of only resorting to T. Kuhn, to paradigm shifts and scientific revolutions [4], in this case, it is possible to refer the reader to a recent book by P. Mirowski: Never Let a Serious Crisis Go to Waste [5]. It is an early account of the failure of "heterodox" economists (including econophysicists) to become relevant for policy making, but it is written by a historian! Let me quote the book blurb in the UK paperback edition verbatim.

At the onset of the Great Recession, as house prices sank and joblessness soared, many commentators concluded that the economic convictions behind the disaster would now be consigned to history. Yet in the harsh light of a new day, attacks against government intervention and the global drive for austerity are as strong as ever. Never Let a Serious Crisis Go to Waste is the definitive account of the wreckage of what passes for economic thought, and how neoliberal ideas were used to solve the very crisis they had created.

In my opinion, as econophysicists, we have lost an important battle against the mainstream in Economics, but not necessarily the war. Even if I doubt that I will soon see

\footnotetext{
a e-mail: e.scalas@sussex.ac.uk
} 
the theory outlined below taught in Economics-101 courses instead of standard microeconomics, we can still make a difference. First of all, whereas economists usually crave to become advisers of the Prince, we are not necessary interested in an immediate socio-economic impact of our ideas. This is indeed a good recipe to avoid disasters. We should look both for reasonable theoretical constructions and for sound empirical validation/falsification of our models. Building a reasonable theory or model can be relatively easy. Corroborating or falsifying the very same theory or model is exremely difficult. For this reson, we must refine and improve our statistical tools. Several of our celebrated empirical finding (a.k.a. stylised facts) would not stand serious criticism by statisticians. Incidentally, this applies to all our empirical works, mine included. In plain words, among other things, this means fewer power laws and more economic insight. Finally, let me mention the two areas where I think econophysics has made a difference so far. The first area is the empirical and theoretical analysis of high frequency data in regulated financial markets. I will not cover this here. The second and, more original, contribution is the (re)introduction of random exchange models applied to the distribution of wealth. On this topic, I will base the next sections. This paper is based on the talk I gave in 2015 at a conference in Ontario mentioned below.

\section{Random exchange models for wealth inequality}

As mentioned in the introduction, one of the most promising lines of research in Econophysics concerns random exchange models for the wealth distribution. Random exchange models were introduced in Econophysics by a paper by Drăgulescu and Yakovenko [6]. However, as remarked by Lux [7], they were already known in the Sociology literature as in the case of the Inequality Process by J. Angle [8]. In Economics, it is possible to find a trace of these processes in the literature in Italian (see [9]). The deep connection between these models and Statistical Physics was explored in a (rather neglected) book by U. Garibaldi and myself [10]. Below, I will provide a general idea on such a connection. The relevant Econophysics and Mathematics literature was reviewed by A.S. Chakrabarti and B.K. Chakrabarti [11]. Recently, five years after the synthesis attempted in [10], I was glad to see that research on these models is quite active. At the Econophysics Colloquium held in Prague in 2015, there was a session devoted to models for the wealth distribution and, with B. Düring, I organised a special session of the AMMCS-CAIMS conference in Waterloo, Ontario devoted to Wealth Distribution and Statistical Equilibrium in Economics (see http://www . ammcs-caims2015.wlu.ca/special-sessions/wdsee/). A new paper came out of this session dealing with the relationship between random exchange models and kinetic theory and is now available [12]. Both sessions were really very interesting and inspiring. It is my hope that the recent success of Piketty's book [13] will help in keeping empirical and theoretical research on the wealth distribution active and in good shape. For random exchange models to be meaningful, it is sufficient to assume that transactions do not occur at economic equilibrium. On the contrary, due to information asymmetry, a part of the wealth of one of the transacting counterparties is transferred to the other counterparty. Even if the simple models outlined below consider wealth as a positive random variable, indebtedness can be taken into account, for example by additively rescaling negative wealth. Finally, if the total wealth is not conserved in the period of interest, this can be taken into account as well, by coupling the global dynamics of wealth with random exchange models. If we believe that random exchange captures the essence of economic transactions, we can see that economic inequality already arises as a consequence of mere chance, even if, in this random case, wealth distributions are not as skewed and unfair as those empirically observed in human societies. However, several variants exist of random 
exchange models that can better reproduce the heavy-tailed distributions observed in reality as discussed in [10] and in [14,15].

The prototypical example of random exchange model is the microcanonical fluid of hard spheres where particles exchange energy as a consequence of elastic collisions. In a microcanonical fluid of hard spheres, the total number of particles $N$ is conserved and the total energy $E$ is conserved. If $E_{i}$ denotes the energy of particle $i$, the normalised particle energies $\varepsilon_{i}=E_{i} / E$ follow a Dirichlet distribution with density:

$$
f_{\boldsymbol{\varepsilon}}(\mathbf{u})=\frac{\Gamma(d N / 2)}{[\Gamma(d / 2)]^{N}} \prod_{i=1}^{N} x_{i}^{d / 2-1} \mathbb{I}_{S}(\mathbf{u})
$$

where $\mathbb{I}_{S}(\cdot)$ is the indicator function of the simplex $S$ defined by $\sum_{i=1}^{N} \varepsilon_{i}=1$ (see [16]). Particles are exchangeable. After marginalising the Dirichlet distribution, one finds that the normalised energy of a single particle follows a Beta distribution with density:

$$
f_{\varepsilon}(u)=\frac{\Gamma(d N / 2)}{\Gamma(d / 2) \Gamma(d(N-1) / 2)} u^{d / 2-1}(1-u)^{d(N-1) / 2-1} \mathbb{I}_{[0,1]}(u) .
$$

Energy is the analogous of wealth and for large $N$ we get a skewed distribution of energy.

The Ehrenfest-Brillouin model studied in [10] is a Markov chain in which $n$ objects can move across $g$ categories according to the following transition probability

$$
\mathbb{P}\left(\mathbf{n}_{i}^{k} \mid \mathbf{n}\right)=\frac{n_{i}}{n} \frac{\alpha_{k}+n_{k}-\delta_{k . i}}{\alpha+n-1}
$$

where the $\alpha_{i}$ s are category weights such that $\sum_{i=1}^{g} \alpha_{i}=\alpha$. The invariant distribution which is also an equilibrium distribution (this is an aperiodic and irreducible Markov chain) is a generalised $g$-dimensional Pólya distribution

$$
\pi(\mathbf{n})=\frac{n !}{\alpha^{[n]}} \prod_{i=1}^{g} \frac{\alpha_{i}^{\left[n_{i}\right]}}{n_{i} !}
$$

where $\alpha^{[n]}=\alpha(\alpha+1) \cdots(\alpha+n-1)$. This was used as a toy model for taxation and redistribution in [17]. Assume that all the $\alpha_{i}=\theta$ for every $i$. The marginal distribution on a category is

$$
\pi(k)=\frac{n !}{k !(n-k) !} \frac{\theta^{[k]}((n-1) \theta)^{[n-k]}}{(n \theta)^{[n]}}
$$

whose continuum limit is (for $u=k / n$ with $k<n$ and $k$ and $n$ large) the density

$$
\pi(u)=\frac{\Gamma(n \theta)}{\Gamma(\theta) \Gamma((n-1) \theta)} u^{\theta-1}(1-u)^{(n-1) \theta-1} \mathbb{I}_{[0,1]}(u) .
$$

The identification $\theta=d / 2$ and $n=N$ gives the same distribution as for the normalised energies in the hard-sphere fluid! 
It is possible to obtain rigorous results on the model originally presented in [9] and later rediscovered in [6]. We consider $g$ agents with $n$ coins distributed among them. Then, the following game is played:

1. At each step a loser is selected by chance from all the agents with at least one coin;

2. the loser gives one of his/her coins to a winner randomly selected among all the agents.

This can be represented by the following transition probability $[18,19]$

$$
\mathbb{P}\left(\mathbf{n}^{\prime} \mid \mathbf{n}\right)=\frac{1-\delta_{n_{i}, 0}}{g-z_{0}(\mathbf{n})} \frac{1}{g},
$$

where $z_{0}(\mathbf{n})$ represents the number of agents without coins. Again, this is an irreducible and aperiodic Markov chain. The invariant and equilibrium distribution is

$$
\pi(\mathbf{n})=C\left(g-z_{0}(\mathbf{n})\right) .
$$

Here, the marginalisation is not trivial even if there is agent exchangeability (see $[18,19]$ for more details). Consider the partition vector $\mathbf{Z}=\left(Z_{0}, \ldots, Z_{n}\right)$ where $Z_{0}$ represents the number of agents with zero coins, $Z_{1}$ the number of agents with one coin, and so on, with $\sum_{i=1}^{n} Z_{i}=g$ and $\sum_{i=1}^{n} i Z_{i}=n$. Naive maximum entropy cannot be used to find the most probable value of $\mathbf{Z}(\pi(\mathbf{n})$ is not uniform), but the multivariate distribution of $\mathbf{Z}$ is available:

$$
\mathbb{P}(\mathbf{Z}=\mathbf{z})=\frac{g !}{z_{0} ! z_{1} ! \cdots z_{n} !} \pi(\mathbf{n})=\frac{g !}{z_{0} ! z_{1} ! \cdots z_{n} !} C\left(g-z_{0}(\mathbf{n})\right) .
$$

The normalization constant $C$ is given by

$$
C=\left[\sum_{k=1}^{g} k\left(\begin{array}{l}
n \\
k
\end{array}\right)\left(\begin{array}{l}
n-1 \\
k-1
\end{array}\right)\right]^{-1} .
$$

One can find $\mathbb{E}\left(Z_{i}\right)$ as

$$
\mathbb{E}\left(Z_{i}\right)=\sum_{k=0}^{g} \mathbb{E}\left(Z_{i} \mid k\right) \mathbb{P}(k)=\sum_{k=0}^{g} g \mathbb{P}\left(n_{1}=i \mid k\right) \mathbb{P}(k), k=g-z_{0},
$$

where

$$
\mathbb{P}(k)=C k\left(\begin{array}{l}
n \\
k
\end{array}\right)\left(\begin{array}{l}
n-1 \\
k-1
\end{array}\right)
$$

and the different values of $\mathbb{E}\left(Z_{i} \mid k\right)$ are given by

$$
\begin{gathered}
\mathbb{E}\left(Z_{0} \mid k\right)=g-k \\
\mathbb{E}\left(Z_{i} \mid k>1\right)=k \frac{\left(\begin{array}{c}
n-i-1 \\
k-2
\end{array}\right)}{\left(\begin{array}{l}
n-1 \\
k-1
\end{array}\right)}, i=1, \ldots, n-1 \\
\mathbb{E}\left(Z_{i} \mid k=1\right)=\delta_{i, n}, i=1, \ldots, n \\
\mathbb{E}\left(Z_{i} \mid k\right)=0, \text { for } n-i-1<k-2 \text { and } i=n .
\end{gathered}
$$


Only for $n \gg g \gg 1$, one gets

$$
\frac{\mathbb{E}\left(Z_{i}\right)}{g} \approx \frac{g}{n}\left(1-\frac{g}{n}\right)^{i},
$$

a geometric distribution coinciding with the naive maximum entropy solution. In fact, in this limit, the probability of finding agents without coins is negligible.

\section{In- and out-of-equilibrium}

In 2010, I wrote a short comment on statistical equilibrium in Economics, which is still available on-line [20] in the site of the Econophysics Forum. I was not aware that the organisers of the site were going to apply for a European Coordination and Support Action entitled Non-Equilibrium Social Science in ICT and Economics which was later funded [21]. An interesting reply to my post came from J.-P. Bouchaud [22] who, among other things, pointed to the role of meta-stability in Economics. I take this opportunity to stress that a useful concept of non-equilibrium immediately emerges from the concept of statistical equilibrium outlined above. In the end, many models used in Economics, both orthodox and heterodox, are Markov chains. Under suitable conditions, these chains admit a unique invariant measure that is also an equilibrium measure. Many techniques are available to study the convergence to equilibrium and hence disequilibrium. Thanks to these techniques, one can even find rigorous upper and lower bounds for mixing times (the times of convergence to equilibrium).

\section{Outlook}

It is difficult to say now what will happen to Econophysics in the future. Judging from the last edition of the Econophysics Colloquium I attended, the discipline seems alive and in relative good shape. Also its future influence on Economics is not clear at this stage. However, it is likely that some tools that econophysicists are using, such as agent-based simulations, will become standard. We must be aware that the time frame of scientific progress is of decades if not centuries. Our work could just be remebered on a sidenote or become of central interest, depending on contingent events.

As a final remark, I would like to mention that I am currently working with B. Düring and N. Georgiou on a paper (mentioned above) where our aim is to show the connection between different methods and models that were used to study random exchange model. I presented this paper at a recent conference in Japan in a session to commemorate my friend and collaborator Jun-ichi Inoue (see [23]). A first version of this paper is now available [12].

\section{References}

1. H.P. Minsky, Stabilizing an Unstable Economy (McGraw-Hill, New York NY, 2008)

2. E. Farjoun, M. Machover, Laws of Chaos: A Probabilistic Approach to Political Economy (Verso, London, 1983)

3. See http://reykjavikmanifesto.wikidot.com and http://reykjavikmanifesto. wikidot.com/statistical-equilibrium-in-economics for my tutorial contribution on statistical equilibrium in Economics

4. T. Kuhn, The Structure of Scientific Revolutions (University of Chicago Press, Chicago IL, 1962) 
5. P. Mirowski, Never Let a Serious Crisis Go to Waste. How Neoliberalism Survived the Financial Meltdown (Verso, London UK, 2014)

6. A.A. Drăgulescu, V.M. Yakovenko, Eur. Phys. J. B 17, 723 (2000)

7. T. Lux, Emergent statistical wealth distributions in simple monetary exchange models: A critical review. In Chatterjee A., S. Yarlagadda, and B.K. Chakrabarti (Eds.), Econophysics of wealth distributions (Springer-Verlag, Milan, 2010)

8. J. Angle, Social Forces 65, 293 (1986)

9. E. Bennati, Rivista Internazionale di Scienze Economiche e Commerciali 8, 735 (1988)

10. U. Garibaldi, E. Scalas Finitary Probabilistic Methods in Econophysics (Cambridge University Press, Cambridge UK, 2010)

11. A.S. Chakrabarti, B.K. Chakrabarti, Economics: The Open-Access, Open-Assessment E-Journal 4, 2010-4 (2010)

12. B. Düring, N. Georgiou, E. Scalas, Stylized model for wealth distribution, arXiv: 1609.08978 (2016)

13. T. Piketty Capital in the Twenty-First Century (Harvard University Press, Cambridge MA, 2014)

14. A. Chatterjee, S. Sinha, B.K. Chakrabarti, Current Science 92, 1383 (2007)

15. S. Sinha, A. Chatterjee, A. Chakraborti, B.K. Chakrabarti, Econophysics: An Introduction (Wiley-VCH, Weinheim, 2010)

16. E. Scalas, A. Gabriel, E. Martin, G. Germano, Phys. Rev. E 92, 022140 (2015)

17. U. Garibaldi, E. Scalas, P. Viarengo, Eur. Phys. J. B 60, 241 (2007)

18. E. Scalas, U. Garibaldi, S. Donadio, Eur. Phys. J. B 53, 267 (2006)

19. E. Scalas, U. Garibaldi, S. Donadio, Erratum: Eur. Phys. J. B 60, 271 (2006)

20. See http://www3. unifr.ch/econophysics/?q=content/case-statisticalequilibrium-economics

21. See http://cordis.europa.eu/project/rcn/102344_en.html

22. See http://www3.unifr.ch/econophysics/?q=content/case-non-equilibriumeconomics

23. See http://c-faculty.chuo-u.ac.jp/ jafee20/inoue.html

Open Access This is an Open Access article distributed under the terms of the Creative Commons Attribution License (http://creativecommons.org/licenses/by/4.0), which permits unrestricted use, distribution, and reproduction in any medium, provided the original work is properly cited. 